healthy controls. Advanced echocardiographic techniques were used to measure GLS, LV E/e' septal and LV E/e' lateral and RV E'/A' in both patients and controls and compare with the parameters measured by conventional echo. Additionally, the CD patients were classified in to 2 groups based on their diet and their cardiac functions were compared accordingly.

Results LVE/e' was significantly higher in CD than controls, $\mathrm{p}$ value $<0.0001$, suggesting diastolic dysfunction in cases compared to controls (table 1). In contrast, LV GLS (\%) was significantly lower in cases compared to controls with pvalue $<0.0001$, suggestive of subtle LV systolic dysfunction in cases with celiac disease. Moreover, both RV GLS (\%) and RV E'/A' were statistically lower CD patients than controls) denoting impaired both RV systolic and RV diastolic functions respectively. Finally, the LVEDV (ml) was lower in patients who were on gluten free diet for $\geq 6$ months Suggestive of positive reverse remodeling of the $\mathrm{LV}$ in celiac disease after implementation of gluten free diet.

\begin{tabular}{llll}
\multicolumn{4}{l}{ Abstract } \\
\hline Variable & $\begin{array}{l}\text { gluten-free diet for }<6 \\
\text { months }(n=14) \text { mean } \pm \text { SD }\end{array}$ & $\begin{array}{l}\text { gluten-free diet for } \geq 6 \\
\text { months }(n=18) \text { mean } \pm \text { SD }\end{array}$ & P-value* $^{*}$ \\
\hline LVEDV (ml) & $78.89 \pm 4.07$ & $72.71 \pm 4.86$ & 0.001 \\
\hline
\end{tabular}

Conclusions Subclinical myocardial dysfunction of both ventricles occurs in children with CD. This observed dysfunction seems to follow the same pattern of improvement of the gut pathology when gluten free diet is implemented.

\section{G344(P) VANCOMYCIN-INDUCED ACUTE KIDNEY INJURY IN NON-CRITICALLY ILL CHILDREN AT A TERTIARY PAEDIATRIC HOSPITAL}

${ }^{1} \mathrm{DE}$ Redding, ${ }^{2,3} \mathrm{~S} J$ McWilliam. 'Medical School, University of Liverpool, Liverpool, UK; ${ }^{2}$ Department of Women's and Children's Health, University of Liverpool, Liverpool, UK; ${ }^{3}$ MRC Centre for Drug Safety Science, University of Liverpool, Liverpool, UK

\subsection{6/archdischild-2020-rcpch.296}

Aims Vancomycin is known to have potential to cause nephrotoxicity. This retrospective audit aimed to describe the epidemiology of acute kidney injury (AKI) in non-critically ill paediatric patients exposed to treatment with intravenous vancomycin, at a tertiary paediatric hospital in the UK.

Methods The electronic medical records of all inpatients at a tertiary paediatric hospital who had one or more vancomycin drug levels measured between 1st October 2018 and 31st March 2019 were reviewed. All patients who received a course of intravenous vancomycin outside of the paediatric intensive care unit were included. Vancomycin-induced AKI was defined as any stage of AKI by the KDIGO criteria (an increase in serum creatinine of more than 50\% from baseline) occurring during, or in the 7 days following, vancomycin exposure.

Results Over six months, 74 courses of intravenous vancomycin were administered to a total of 60 children. There were 12 episodes of vancomycin-induced AKI in 12 children (7 $(58.3 \%)$ stage $1,3(25 \%)$ stage $2,2(16.7 \%)$ stage 3$)$. The incidence of vancomycin-induced AKI was 16.2 per 100 courses and 20 per 100 children.

\section{Abstract G344(P) Table 1}

\begin{tabular}{lll}
\hline & No AKI (n=62) & AKI ( $\mathbf{n = 1 2 )}$ \\
\hline Age (mean, SD) & $2.65(4.99)$ & $7.66(5.75)$ \\
Male (N,\%) & $39(62.9 \%)$ & $7(58.3 \%)$ \\
Concomitant nephrotoxins (mean, SD) & $0.71(0.86)$ & $1.58(1.24)$ \\
Trough >20 (N,\%) & $8(12.9 \%)$ & $2(16.7 \%)$ \\
Days of Vancomycin (Mean, SD) & $4.13(2.24)$ & $4.5(4.17)$ \\
Initial Vancomycin dose (mg/kg/day) (mean, SD) & $24.33(12.86)$ & $25.85(7.62)$ \\
Peak Vancomycin dose (mg/kg/day) (mean, SD) & $37.55(17.89)$ & $41.71(17.72)$ \\
Previous renal disease (N,\%) & $12(19.4)$ & $2(16.7 \%)$ \\
Previous AKI (N,\%) & $6(9.7 \%)$ & $5(41.7 \%)$ \\
Previous cardiac bypass (N,\%) & $16(25.8 \%)$ & $4(33.3 \%)$ \\
\hline
\end{tabular}

Concomitant nephrotoxin exposure was greater in the AKI group (table 1). The most frequent concomitant nephrotoxins associated with vancomycin-induced AKI were diuretics (furosemide and spironolactone) $(n=6,50 \%)$, piperacillin-tazobac$\operatorname{tam}(n=3,25 \%)$ and teicoplanin $(n=3,25 \%)$.

Conclusion Incidence of vancomycin-induced acute kidney injury in children is high, as seen in other studies. Patients on vancomycin should receive close monitoring for the development of AKI, including daily measurement of serum creatinine. Modifiable risk factors, such as exposure to concomitant nephrotoxins, should be minimised.

\section{G345(P) ABSTRACT WITHDRAWN}

\section{G346(P) ABSTRACT WITHDRAWN}

\section{G348(P) URSODEOXYCHOLIC ACID IMPROVES METABOLIC OUTCOMES IN THE FETUS, PLACENTA AND OFFSPRING OF HYPERCHOLANEMIC PREGNANCY}

${ }^{1} \mathrm{~A}$ Qadri, ${ }^{2} \mathrm{G}$ Papacleovoulou, ${ }^{2} \mathrm{LB}$ Manna, ${ }^{2} \mathrm{~V}$ Formigo-Pataia, ${ }^{2} \mathrm{~S}$ Abu-Hayyeh, ${ }^{2} \mathrm{P}$ Dixon, ${ }^{2}$ F Flaviani, ${ }^{2} \mathrm{C}$ Williamson. ${ }^{1}$ Institute of Reproductive and Developmental Biology, Imperial College London, London, UK; ${ }^{2}$ Maternal and Fetal Disease Group, Division of Women's Health, Kings College London, London, UK

\subsection{6/archdischild-2020-rcpch.297}

Aims Perturbations in the intrauterine environment can result in lifelong consequences for fetal metabolic health. We previously showed that in humans, intrahepatic cholestasis of pregnancy (ICP) can predispose offspring to metabolic disease, likely due to a combination of the effects of increased bile acids (BA), maternal dyslipidemia and deranged maternal and fetal lipid homeostasis. Ursodeoxycholic acid (UDCA) is a well-established treatment for maternal ICP. We aimed to address whether UDCA can also prevent the adverse metabolic effects of ICP in the offspring and fetoplacental unit.

Methods Women with ICP \pm UDCA treatment and controls with uncomplicated pregnancy were recruited for epigenome wide association studies which were performed on maternal and venous umbilical cord blood. Animal studies 